\title{
The microbial physiologist's guide to the leprosy genome
}

\author{
PAUL R. WHEELER \\ Tuberculosis Research Unit, Veterinary Laboratories Agency \\ Weybridge, New Haw, Surrey, UK, KT15 3NB, UK
}

\section{Introduction}

One of the most exciting things about living in the genome sequencing era is that hitherto intractable pathogens can be understood for the first time in unimaginable detail. Many bacterial genomes have been sequenced and one can now enquire about their physiology. It is possible not just to enquire about Mycobacterium leprae, but make comparisons with other host-dependent and axenically uncultivable pathogens ${ }^{1}$ such as Treponema pallidum, that have also had their genomes sequenced.

Previously, to study the metabolism and biochemistry of leprosy bacilli one had to purify them from the heavily infected tissues of armadillos or nude mice. Some data were obtained from less heavily infected sources including even human biopsies but from such limited material only very limited data could be obtained. To be able to start to deduce metabolic pathways in operation, greater numbers of leprosy bacilli from more heavily infected tissue were needed. ${ }^{2}$ Regardless of the source, it was always essential to prove that any metabolic activity was from the bacteria, and not contamination from the host. For example, catalase activity is readily detected in suspensions and extracts of leprosy bacilli but biochemical ${ }^{3}$ and genetic $^{4,5}$ analysis show that this was host-derived, and there was no mycobacterial KatG (see Box 1) catalase activity. All this was difficult, but perhaps on balance easier than having to extract bacteria from rabbit testicles as is the case for the aforementioned agent of syphilis, T. pallidum.

Now there is genome data to mine. The truth is in there, or rather predictions of the truth are. It is important to keep in mind that deducing a function for a gene (that is, to give an example, referring to ML2323 as ask, the gene for aspartokinase) are predictions of function made on the basis of similarities to raw sequence information elsewhere in nature. Many of the similarities are so high, and so consistent throughout a metabolic pathway, that the attributions are essentially incontestable. These have been compiled into metabolic pathways in the KEGG database (http://www.genome.ad.jp/kegg/) where, for example, the nucleotide biosynthetic pathways referred to in Box 1 can be viewed. Other areas of metabolism are still 
Box 1. The physiology of Mycobacterium leprae as deduced from its genome

- Uniquely for a host-dependent pathogen, the biosynthetic pathways for purine and pyrimidine nucleotides are complete.

- Biosynthetic pathways for lipids and amino acids are also complete with the exception that M. leprae is a methionine auxotroph.

- Only central pathways of carbon and energy metabolism are complete but alternative pathways are degenerate.

- The co-enzyme central to the most universal metabolic pathways - NADH cannot be recycled to NAD by the usual oxidative respiratory route.

- Redundancy seen in $M$. tuberculosis is often lost in $M$. leprae, as most paralogues seen in M. tuberculosis are pseudogenes (= non-functional) in M. leprae.

- Defence against toxic radicals is severely degenerate, as neither katG nor the narGHJl cluster is functional.

- None of the few (142) additional genes only found in $M$. leprae appear to confer additional metabolic pathways.

far from clear. Some of these are explored in this review, such as whether M. leprae is capable of the biosynthesis of siderophores for the vital activity of acquisition of iron from the environment. This is but one example of how the genomic data generates hypotheses to be tested. There are yet others to be generated. The 'take-home message' is that the genome sequence is not the conclusion of research into any particular microbe, but merely the 'end of the beginning', a beginning started with rational research on microbial metabolism and the action of antimicrobial agents by Pasteur, Entner and Doudoroff, Stamp and Green amongst others. $^{6}$

\section{Anabolic and catabolic pathways - what's different about M. leprae?}

The big questions in bacterial physiology are: how do bacteria obtain their nutrition, use it for production of energy (catabolism) and make all their components - proteins, lipids, cell envelope, nucleic acids and so on (anabolism). The first four points in Box 1 summarize how the genome project has shown how $M$. leprae differs from other microbes in general, while the fifth point shows how M. leprae differs from Mycobacterium tuberculosis in particular.

Microbes of medical interest vary considerably in their metabolic capabilities. At one extreme, some are autotrophs so that they can make everything they need from ammonium ions and carbon dioxide. At the other extreme, there are highly host-dependent organisms such as Rickettsia and treponeme species. ${ }^{1}$ A classical example of their dependency upon the host for preformed intermediates is that they are unable to synthesize the structures on which nucleotides are based - purine and pyrimidine rings that include four to five carbon atoms and two to five nitrogen atoms. Thus, like many parasitic microbes, they have to scavenge purines and pyrimidines from their host. Intuitively, M. leprae would be expected to lie on the hostdependent end of this scale, since it cannot be grown axenically and it grows almost by stealth when it is in the host, dividing no more rapidly than every 12 days (growth rate $\left.\sim 0.0035 \mathrm{~h}^{-1}\right){ }^{7}$ However, a surprising finding from the genome sequence is that M. leprae has the capacity to make most of its own components. For example, although it is able to obtain lipids, amino acids, purines and pyrimidines directly from the host, ${ }^{4}$ it can also make all these from the simplest components. In this respect its metabolism is no different from $E$. coli or M. tuberculosis. The one exception is that M. leprae cannot make methionine and must 
Table 1. Distribution of genes by selected functions

\begin{tabular}{lccc}
\hline Category & $\begin{array}{c}\text { M. tuberculosis } \\
\text { genes }\end{array}$ & $\begin{array}{c}\text { M. leprae } \\
\text { genes }\end{array}$ & $\begin{array}{c}\text { M. leprae } \\
\text { pseudogenes }\end{array}$ \\
\hline $\begin{array}{l}\text { Energy metabolism } \\
\text { Nucleotide and nucleoside metabolism }\end{array} \quad 300$ & 95 & 120 \\
$\quad$ (includes purine and pyrimidine biosynthesis) & 65 & 57 & 5 \\
Amino acid biosynthesis & 91 & 78 & 5 \\
Lipid biosynthesis & 68 & 37 & 12 \\
VE/PPE & 167 & 8 & 8 \\
Virulence determinants & 39 & 16 & 4 \\
\hline
\end{tabular}

depend upon the host for this one amino acid. This one piece of degeneracy in the major anabolic pathways is evidently something that pathogenic mycobacteria can tolerate, since experimentally obtained methionine auxotrophs of $M$. tuberculosis show little or no attenuation. $^{8,9}$

Compare now in Table 1 the difference between the distribution of genes for the anabolic pathways I have just mentioned and those for energy metabolism. The former are virtually complete, with losses in $M$. leprae compared with $M$. tuberculosis being down to loss of redundancy more often than function. Thus, whereas $M$. tuberculosis may have two or more genes capable of performing a particular function, $M$. le prae frequently has just one. Even in the case of lipid biosynthesis, central pathways are complete with the losses reflecting the more limited, but still extensive, repertoire of lipids produced by $M$. leprae. However, for energy metabolism (catabolism) $M$. leprae has less than one-third the functional genes possessed by $M$. tuberculosis. Here, alternative pathways are lost and $M$. leprae has a very limited aerobic respiratory metabolism. It is possible for $M$. leprae to use glucose or glycerol or even pyruvate, catabolizing them to carbon dioxide to generate ATP oxidatively (Figure 1). However, the rate at which pyruvate is converted to acetyl-CoA to feed carbon into the oxidative Krebs cycle (Figure 1) is limited by a loss of redundancy. The $p d h$ genes found in M. tuberculosis are lost in M. leprae and the function - the corresponding enzymatic activity - is markedly low. ${ }^{10}$ This remaining function is apparently encoded by aceE and $l p d$, two separate genes that are ML1651 and ML2387, respectively. Disregarding the rate of catabolism of glucose and glycerol to carbon dioxide, their oxidation is predicted to generate only about $35 \%$ the ATP per mole carbon source oxidized that analogous pathways in M. tuberculosis generate, since all the NAD is predicted to be recycled in an oxygenindependent way (Figure 1). This failure to couple NADH oxidation to electron transport appears to be an adaptation to low oxygen tension yet the anaerobic respiratory systems seen in M. tuberculosis are all gone in M. leprae. Finally, the inability to recycle acetate (Figure 1) is predicted to lessen opportunities for axenic growth by limiting the number of carbon sources that $M$. leprae can use. ${ }^{11}$

Other notable differences between $M$. leprae and M. tuberculosis occur in the anaplerotic pathways in which 3-carbon (pyruvate, phospho-enol-pyruvate or PEP) and 4-carbon compounds (e.g. oxaloacetate, malate) can be interconverted to be used in either a catabolic or anabolic direction (Figure 1). Here, $M$. leprae lacks the pca gene. This is predicted to be required for pyruvate to be used in gluconeogenesis in groups of organisms that posses $p c a$, such as actinomycetes and yeasts; thus another component of carbon metabolism is lacking in 


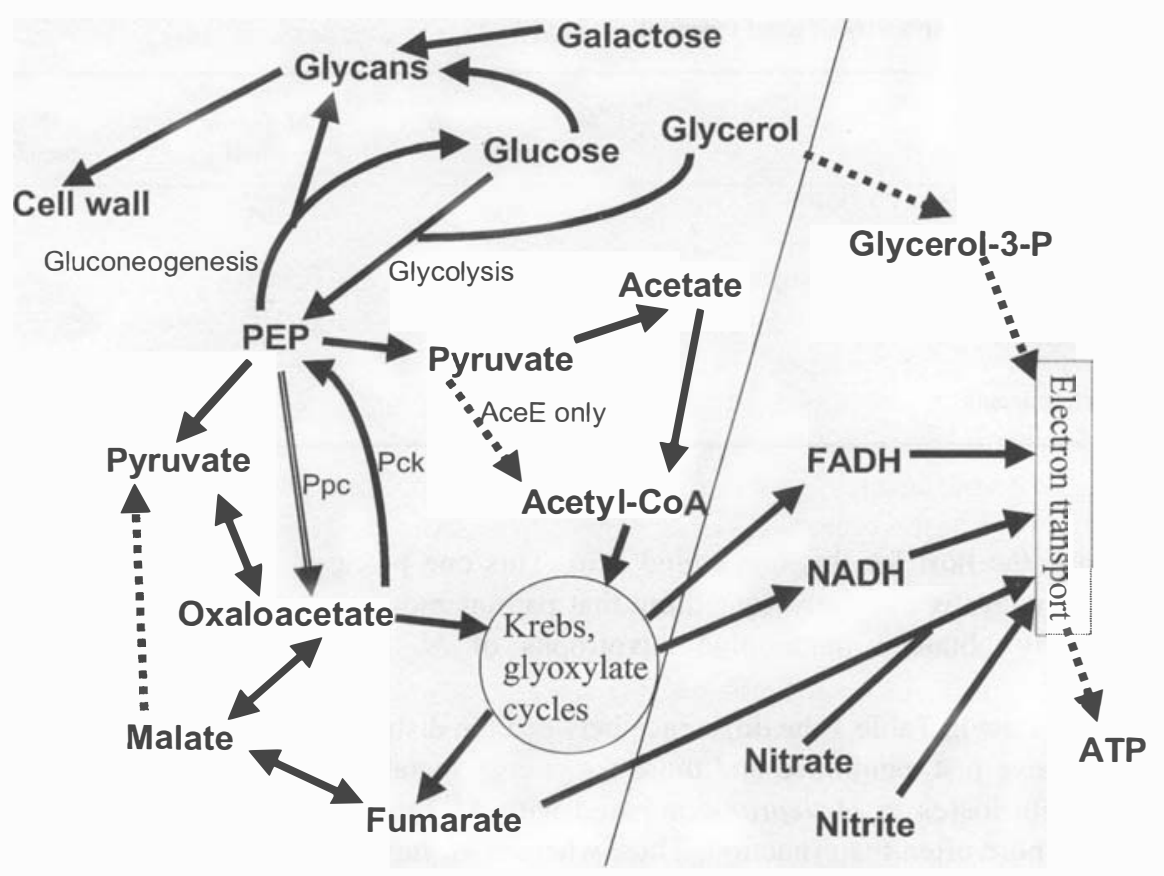

Figure 1. Carbon and energy metabolism in M. leprae; a comparison with a pathogen that can be cultured on simple media, $M$. tuberculosis. This simplified figure shows how glucose, glycerol, pyruvate, acetate and galactose can be metabolized in leprosy bacteria. Black arrows show reactions that can take place in both bacteria, gray ones only in $M$. tuberculosis and the open arrow shows the reaction that can only occur in M. leprae. Dashed arrows are for reactions predicted to occur at a limited rate. Left of the line arrows show interconversion of substrates, right of the line arrows show electron flow, in essentially respiratory pathways. The conversion of PEP (phospho-enol-pyruvate) to pyruvate is shown twice for clarity. For M. leprae, it follows that glucose and glycerol can be used for both ATP generation and gluconeogenesis to lead to cell wall intermediates as black arrows can be followed throughout the pathways. Pyruvate can only be used to generate ATP in the pathways shown in this figure, and acetate and galactose cannot be used at all. All substrates can be used in all directions by tubercle bacilli.

M. leprae. Intriguingly, M. leprae has a gene not present in M. tuberculosis, ppc (Figure 1). It is hard to predict the function of $p p c$, with certainty. Possibly it would allow oxaloacetate to be topped up so that the citric acid cycle could continue through dismutation of PEP (Figure 1). The effects of these differences should be ascertained by comparing the metabolic fate of pyruvate, glycerol, and perhaps other carbon sources (see Figure 1 for examples) in M. leprae and other actinomycetes.

A further enzyme in interconverting 3- and 4-carbon compounds is malic enzyme (decarboxylating), which converts malate to pyruvate. The dichotomy between fast and slow growing mycobacteria was once thought to be based, with few exceptions, on the presence or absence of this enzyme, with its activity found only in fast growers (mycobacteria that have a growth rate of above about $0.15 \mathrm{~h}^{-1}$ ). Intriguingly the corresponding gene, $m e z$, is actually found in $M$. tuberculosis ( $\mathrm{Rv} 2332)$, but is a pseudogene in M. leprae. Together with the apparent 'pinch-point' for conversion of pyruvate to acetyl-CoA mentioned above, this may reflect an irreversible commitment of $M$. leprae to a lifestyle characterized by slow growth and necessarily slow central metabolism.

The overall conclusion is that loss of alternative pathways in catabolism and also the loss 
of 'redundant' genes (Box 1) has resulted in $M$. leprae occupying, and being severely restricted to a specialized niche. But there is worse to come!

\section{Defence against toxic radicals}

Intracellular pathogens such as $M$. leprae and $M$. tuberculosis have to protect themselves from exposure to toxic products produced by host macrophages. $M$. leprae lacks major components of its defences against the highly toxic and damaging oxygen- and nitrogen-free radicals. Against oxygen radicals there is no functional kat $G$ to encode catalase. This is evident from the genome sequence and has been proved biochemically ${ }^{3}$ and genetically. ${ }^{4,5}$ The narGHJI gene cluster, ${ }^{12}$ which is lacking in $M$. leprae, may be a virulence factor, and could be involved in resistance against nitrogen oxide radicals. Their deletion, together with the lack of a functional $k a t G$ to encode catalase as a defence against oxygen-free radicals, makes $M$. leprae look very vulnerable to the host's cell defence mechanisms. This reduction in the genes for respiration and to deal with the consequences of an oxygen-rich environment may therefore reflect a niche with relatively constant conditions (intracellular, little toxicity, a fairly constant microaerophilic environment) for $M$. le prae. In contrast, $M$. tuberculosis exists in a variety of tissues, from intracellular when it has been engulfed by alveolar macrophages, to caseated, probably almost anaerobic, lesions. Extreme specialization of the leprosy bacillus in terms of its oxygen environment may account for the deletion or inactivation in the $M$. leprae genome of 145 of the 170 remaining oxidoreductases and oxygenases found in M. tuberculosis.

\section{Iron acquisition and metabolism}

Classical studies show that the ability to acquire iron from the host is essential for the survival and growth of intracellular pathogens. Yet in comparison with M. tuberculosis M. leprae appears to have a major deficiency in its ability to acquire iron. Its entire $m b t$ operon is deleted, rendering it unable to make either the membrane-associated or excreted form of mycobactin T. In M. tuberculosis, such a deletion would lead to loss of virulence as deletion of the $m b t B$ gene results in restriction of growth in iron-limited media and in macrophages. ${ }^{13}$ However, suspensions of $M$. leprae do not take up iron from the excreted mycobactin siderophores of slow-growing mycobacteria including $M$. tuberculosis and Mycobacterium avium. Instead, they acquire iron from the exochelin siderophores of Mycobacterium neoaurum, one of the most rapid-growing and least fastidious mycobacteria, and an unclassified, cultivable, armadillo-derived mycobacterium. Although they share the function of iron acquisition, these two types of siderophores are chemically distinct. While mycobactin $\mathrm{T}$ is derived from salicylate, the exochelins are hydroxymate-derived siderophores. ${ }^{14}$ Thus, maybe it is the genes for a hydroxymate-based siderophore that should be searched for in the $M$. leprae genome. The genes for the biosynthesis, and probably the export and uptake of the exochelin of $M$. smegmatis are known. ${ }^{15}$ The $f x b$ genes for biosynthesis have no strong matches in the M. leprae or M. tuberculosis genomes even though one would predict that $M$. leprae would synthesize the class of siderophore that it can transport. However, there are important differences between the exochelins of $M$. smegmatis and $M$. neoaurum (for example the use of D-ornithine and $\mathrm{N}$-methylation in the $M$. noeaurum in 
contrast to L-ornithine and N-formylation in M. smegmatis) (see ${ }^{14}$ for a comparison of the full structures) so all the similarity one might expect to see in the genes could be peptide synthases. Thus it is difficult to deduce whether $M$. leprae possesses functional genes for siderophore biosynthesis. However, it might be more feasible to search for genes involved in their transport. In $M$. smegmatis, $f x u D$ is regarded as the gene encoding the ferri-siderophore receptor, but while this has $36 \%$ identity to the $M$. tuberculosis fecB2 gene for an iron transport protein (Rv0265c), the corresponding gene (ML2548) in the M. leprae genome is a pseudogene. A cluster of three orfs immediately downstream of the $M$. smegmatis exochelin biosynthesis locus have strong similarity to clusters of three orfs (in both M. tuberculosis (Rv1458c to Rv1456c) and M. leprae (ML0589 to ML0591) but in both pathogens these appear neither to be under iron regulation nor flanked by genes likely to be involved in iron metabolism.

While the genes involved in iron acquisition by the leprosy bacillus remain obscure, there is no doubt that $M$. leprae does utilize iron. The presence of genes for cytochrome c $(\operatorname{ccs} A, B)$, a haemoglobin-like oxygen carrier $(g l b O)$, ferredoxin $(f d x C, D)$, biosynthesis of the haem group (hem genes) and for the iron-storage bacterioferritin $b f r A$ (in one of the many examples of loss of redundancy, a second, $b f r B$ gene present in $M$. tuberculosis is deleted from $M$. leprae) are all examples of genes encoding iron-containing molecules. Finally, IdeR, the key iron regulation protein dependent on intracellular iron, is highly conserved, with $84 \%$ identity to the M. tuberculosis IdeR. As in M. tuberculosis, ideR is just downstream of another important regulator, $\operatorname{sig} B$, which is involved in response to stress and growth in macrophages. The capacity of $M$. leprae to tightly regulate intracellular iron would be anticipated to be vital since its $k a t G$, the gene that encodes the major catalase activity of pathogenic mycobacteria, is a pseudogene. Thus an excess of iron that would catalyse the formation of peroxides and oxygen-free radicals would be especially disastrous for the leprosy bacillus, and just as harmful to the bacterium as iron deficiency.

\section{Do the genes found only in $M$. leprae explain its neurotropism?}

Two distinctive properties of $M$. leprae are its ability to invade and grow in peripheral nerves, and its production of a unique phenolic glycolipid that forms a capsule around the bacillus. These properties are now thought to be linked, as the phenolic glycolipid binds to peripheral nerves. This lipid binds to the basal lamina of Schwann cell axons in a specific way that is inhibited by both (neural) laminin fragments and the trisaccharide moiety of the glycolipid. ${ }^{16}$ Another product that is involved in binding to Schwann cells is the laminin binding protein, Lbp, though this is found in other mycobacteria. ${ }^{17}$ The phenolic glycolipid is unique because it has a unique trisaccharide moiety, whereas other similar glycolipids, such as the one found in $M$. bovis, have a single sugar. ${ }^{18}$ The genes for adding the additional sugars have not been identified yet, ${ }^{4}$ but the 142 genes specific to $M$. leprae are not candidates since they are too short to encode for the required glycosyltransferases enzymes. However, some of the apparent gene fragments amongst the 142 specific genes could be large enough to encode neuroactive peptides.

Genes found in M. leprae but not in $M$. tuberculosis, for which it has been possible to attribute functions, are shown in Table 2. It is hard to implicate any of them in neurotropism but they do appear to be involved in uptake and metabolism. The adenyl cyclase and nramp homologues (ML2341 and ML2667, respectively) may suggest unusual regulatory functions, 
Table 2. Genes with attributed functions in M. leprae but not in M. tuberculosis

\begin{tabular}{|c|c|c|}
\hline Gene & Function & Paralogues in ... \\
\hline ML0397 & ABC transporter involved in ribose transport? & Bacillus subtilis, other bacteria \\
\hline ML0398 & D-Ribose binding protein & Bacillus subtilis, other bacteria \\
\hline ML0458 & Possible oxidoreductase & $\begin{array}{l}\text { Bacteria and eukaryotes, e.g. } \\
\text { Schizosaccharomyces pombe }\end{array}$ \\
\hline ML0578 & Phospho-enol-pyruvate carboxylase (Ppc) & $\begin{array}{l}\text { Bacteria, e.g. Rhodopseudomonas } \\
\text { palustris and streptomycetes }\end{array}$ \\
\hline $\begin{array}{l}\text { Between ML1092 and } \\
\text { ML1093 }\end{array}$ & Similarities to malate oxidoreductases & Other bacteria \\
\hline ML1553 & ProS, prolyl-tRNA synthetase & Eukaryotic \\
\hline ML1795 & hsp18 & $\begin{array}{l}\text { Other actinomycete hsps, e.g. in } \\
\text { Mycobacterium intracellulare and } \\
\text { Streptomyces albus }\end{array}$ \\
\hline ML2177 & Uridine phosphorylase & Eukaryotic \\
\hline ML2341 & Adenylate cyclase - regulatory function & $\begin{array}{l}\text { Many, e.g. Stigmatella aurantiaca } \\
\text { and Streptomyces coelicolor }\end{array}$ \\
\hline ML2667 & $\begin{array}{l}\mathrm{Mn} 2+\text { transporter, similar to nramp } \\
\text { family - regulatory function }\end{array}$ & $\begin{array}{l}\text { Bacteria, e.g. Pseudomonas } \\
\text { aeruginosa }\end{array}$ \\
\hline
\end{tabular}

perhaps related to a predilection of the leprosy bacillus for adenosine, a property predicted by classical biochemical studies. ${ }^{19}$ A possible link that emerges from the M. leprae genome is that while most of the genes for making cohalamin and related vitamins have been lost in $M$. leprae, most of the few that remain use adenosine or its derivatives as one of their substrates. While the genes for making cobalamin are in $M$. tuberculosis, another of the genes found only in $M$. leprae (uridine phosphorylase) encodes an enzyme in pyrimidine nucleotide metabolism. There is something unusual about this whole area of purine and pyrimidine metabolism in $M$. leprae. Why should the biosynthetic pathways have been retained in such a host-dependent pathogen? Maybe it is part of the mechanism that allows the leprosy bacillus to survive and grow within the rather metabolically inert Schwann cells. Most leprosy bacilli that have been used for biochemical work were obtained from established infections in macrophages (spleens, lymph nodes and livers of experimentally infected armadillos ${ }^{2}$ ). In such established macrophage infections, biochemical studies showed that $M$. leprae only uses scavenging pathways typical of obligate parasites and not the de novo purine biosynthesic pathways $^{20}$ of which it is capable according to its genome. It is possible that the de novo purine biosynthetic pathway is required to establish infection if the early stages are analogous to $M$. tuberculosis infections in which purC mutants in the de novo pathway are cleared by innate immunity. ${ }^{21}$

In this section, I have raised a number of speculative ideas. However, it should be possible to test these ideas in the post-genomic era. It may be possible, for example to compare the transcripts of mRNA produced by leprosy bacilli in macrophages and in Schwann cells. Thus one could identify genes that are preferentially expressed during growth in Schwann cells and hence might account for this unusual property.

\section{A few conclusions and many more questions}

Obtaining a genome sequence should be regarded as a springboard to research on the microbe 
in question. I hope in this short review I have shown that this is a time to push forward with research into $M$. leprae, a paradigm of obligate intracellular host-dependency. Fundamental questions for an intracellular pathogen about iron metabolism can be addressed. How the loss of redundancy throughout the genome in comparison with the tubercle bacilli has resulted in a specialized pathogen in contrast to the adaptable $M$. tuberculosis complex is another basic issue in mycobacteriology. Does the apparent limitation in virulence determinants and cell entry genes (Table 1) commit $M$. leprae to gaining access only to its narrow niche? While two of the three haemolysin genes found in M. tuberculosis, including tlyA, persist in M. leprae, all four plc genes are lost. Will we find out the role of PPEs and PEs in tubercle bacilli by making comparisons with $M$. leprae, a 'natural mutant' for most of them? What else can we learn about tubercle bacilli by comparative genomics now we have the leprosy genome? Why cannot $M$. leprae be grown axenically; do the lesions in energy metabolism only allow interrupted growth when conditions are just right in the host? Are media too toxic, at least in aerobic conditions? With a massive loss of regulatory functions ${ }^{4}$ have those that would allow $M$. leprae to adapt to axenic culture been lost? We are now in a position to generate better defined hypotheses. Soon the Mycobacterium ulcerans genome will be sequenced; will comparisons with this difficult to grow mycobacterium help us to formulate new hypotheses for M. leprae? Finally, we can design experiments that will provide a better understanding of the interaction between $M$. leprae and Schwann cells. With neurological reactions still a major clinical issue in the treatment of leprosy these are urgent experiments. My conclusion would be to recommend the leprosy research community develops post-genomic research and investigates the expression of $M$. leprae genes as a means of addressing the many biological questions that still remain.

\section{References}

${ }^{1}$ Wheeler PR. Understanding the physiology of difficult, pathogenic bacteria from analysis of their genome sequences. J Med Microbiol, 2001; 51: 1-4.

2 Wheeler PR. Recent research into the physiology of Mycobacterium leprae. Adv Microb Physiol, 1990; 31: 71124.

${ }^{3}$ Wheeler PR, Gregory D. Superoxide dismutase, peroxidatic activity and catalase in Mycobacterium leprae purified from armadillo liver. J Gen Microbiol, 1980; 121: 457-464.

4 Cole ST, Eiglmeier K, Parkhill J et al. Massive gene decay in the leprosy bacillus. Nature, 2001; 409: $1007-1011$.

5 Nakata N, Matsuoka M, Kashiwabara Y et al. Nucleotide sequence of the Mycobacterium leprae katG region. J Bacteriol, 1997; 179: 3053-3057.

${ }^{6}$ Beck RW. A chronology of microbiology in historical context. American Society for Microbiology Press, Washington DC, USA, 2000.

7 Levy L. Studies of the mouse foot pad technique for cultivation of Mycobacterium leprae. 3. Doubling time during logarithmic multiplication. Lepr Rev, 1976; 47: 103-106.

8 McAdam RA, Weisbrod TR, Martin J et al. In vivo growth characteristics of leucine and methionine auxotrophic mutants of Mycobacterium bovis BCG generated by transposon mutagenesis. Infect Immun, 1995; 63: 1004-1012.

9 Smith DA, Parish T, Stoker NG, Bancroft GJ. Characterization of auxotrophic mutants of Mycobacterium tuberculosis and their potential as vaccine candidates. Infect Immun, 2001; 69: 1142-1150.

10 Wheeler PR. Oxidation of carbon sources through the tricarboxylic acid cycle in Mycobacterium leprae grown in armadillo liver. J Gen Microbiol, 1984; 130: 381-389.

11 Wheeler PR, Ratledge C. Use of carbon sources for lipid biosynthesis in Mycobacterium leprae: a comparison with other pathogenic mycobacteria. J Gen Microbiol, 1988; 134: 2111-2121.

12 Weber I, Fritz C, Ruttkowski S et al. Anaerobic nitrate reductase (narGHJI) activity of Mycobacterium bovis BCG in vitro and its contribution to virulence in immunodeficient mice. Mol Microbiol, 2000; 35: 1017-1025.

13 De Voss JJ, Rutter K, Schroeder BG et al. The salicylate-derived mycobactin siderophores of Mycobacterium tuberculosis are essential for growth in macrophages. Proc Natl Acad Sci USA, 2000; 97: 1252-1257.

14 Ratledge C. Iron metabolism. In: Ratledge C, Dale J (eds) Mycobacteria. Molecular biology and virulence. Blackwell Science, Oxford, 1999, pp. 260-286. 
15 Yu S, Fiss E, Jacobs WR, Jr. Analysis of the exochelin locus in Mycobacterium smegmatis: biosynthesis genes have homology with genes of the peptide synthetase family. J Bacteriol, 1998; 180: 4676-4685.

${ }^{16} \mathrm{Ng} \mathrm{V}$, Zanazzi G, Timpl R et al. Role of the cell wall phenolic glycolipid-1 in the peripheral nerve predilection of Mycobacterium leprae. Cell, 2000; 103: 511-524.

17 de Melo Marques MA, Mahapatra S, Nandan D et al. Bacterial and host-derived cationic proteins bind alpha2laminins and enhance Mycobacterium leprae attachment to human Schwann cells. Microbes Infect, 2000; 2: $1407-1417$.

18 Dermateau-Ginsburg H, Lederer E. Sur la structure chemique du mycoside B. Biochim Biophys Acta, 1963; 70: 442-451.

19 Wheeler PR. Enzymes for purine synthesis and scavenging in pathogenic mycobacteria and their distribution in Mycobacterium leprae. J Gen Microbiol, 1987; 133: 3013-3018.

20 Wheeler PR. Biosynthesis and scavenging of purines by pathogenic mycobacteria including Mycobacterium leprae. J Gen Microbiol, 1987; 133: 2999-3011.

21 Jackson M, Phalen SW, Lagranderie M et al. Persistence and protective efficacy of a Mycobacterium tuberculosis auxotroph vaccine. Infect Immun, 1999; 67: 2867-2873. 